\section{Acknowledgments}

J.A. Helms is supported in part by the Oak Foundation.

Address correspondence to: Jill A. Helms, Department of Surgery, Stanford University, 257 Campus Drive, GK 207, Stanford, California 94305, USA. Phone: (650) 736-0919; Fax: (650) 736-4374; E-mail: jhelms@stanford.edu.

1. Massa, N. 1536. Liber introductorius anatomiae, sive dissectionis corporis humani. Venetiis. Cap. 21:37-38.

2. Riddle, R.D., Johnson, R.L., Laufer, E., and Tabin, C. 1993. Sonic hedgehog mediates the polarizing activity of the ZPA. Cell. 75:1401-1416.

3. Litingtung, Y., Dahn, R.D., Li, Y., Fallon, J.F., and Chiang, C. 2002. Shh and Gli3 are dispensable for limb skeleton formation but regulate digit number and identity. Nature. 418:979-983.

4. Dahn, R.D., and Fallon, J.F. 2000. Interdigital regulation of digit identity and homeotic transformation by modulated BMP signaling. Science. 289:438-441
5. Colnot, C., et al. 2005. Indian hedgehog synchronizes skeletal angiogenesis and perichondrial maturation with cartilage development. Development. 132:1057-1067.

6. St-Jacques, B., Hammerschmidt, M., and McMahon, A.P. 1999. Indian hedgehog signaling regulates proliferation and differentiation of chondrocytes and is essential for bone formation. Genes Dev. 13:2072-2086.

7. Chung, U.I., Schipani, E., McMahon, A.P., and Kronenberg, H.M. 2001. Indian hedgehog couples chondrogenesis to osteogenesis in endochondral bone development. J. Clin. Invest. 107:295-304.

8. Archer, C.W., Dowthwaite, G.P., and Francis-West, P. 2003. Development of synovial joints. Birth Defects Res. C. Embryo Today. 69:144-155.

9. Hartmann, C., and Tabin, C.J. 2001. Wnt-14 plays a pivotal role in inducing synovial joint formation in the developing appendicular skeleton. Cell. 104:341-351.

10. Niedermaier, M. 2005. An inversion involving the mouse Shh locus results in brachydactyly through dysregulation of Shh expression. J. Clin. Invest. 115:900-909. doi:10.1172/JCI200523675.

11. Vortkamp, A., et al. 1996. Regulation of rate of cartilage differentiation by Indian hedgehog and PTH-related protein. Science. 273:613-622.

12. Hu, D., Marcucio, R.S., and Helms, J.A. 2003. A zone of frontonasal ectoderm regulates patterning and growth in the face. Development. 130:1749-1758.

13. Hu, D., and Helms, J.A. 1999. The role of sonic hedgehog in normal and abnormal craniofacial morphogenesis. Development. 126:4873-4884.

14. Jeong, J., Mao, J., Tenzen, T., Kottmann, A.H., and McMahon, A.P. 2004. Hedgehog signaling in the neural crest cells regulates the patterning and growth of facial primordia. Genes Dev. 18:937-951.

15. Gorlin, R.J., Cohen, M.M., and Levin, L.S. 1990. Syndromes of the head and neck. Oxford University Press. New York, New York, USA. 977 pp.

16. Garciadiego-Cazares, D., Rosales, C., Katoh, M., and Chimal-Monroy, J. 2004. Coordination of chondrocyte differentiation and joint formation by alpha5beta 1 integrin in the developing appendicular skeleton. Development. 131:4735-4742.

17. Dahmann, C., and Basler, K. 2000. Opposing transcriptional outputs of Hedgehog signaling and engrailed control compartmental cell sorting at the Drosophila A/P boundary. Cell. 100:411-422.

18. Sarkar, L., et al. 2000. Wnt/Shh interactions regulate ectodermal boundary formation during mammalian tooth development. Proc. Natl. Acad. Sci. U. S. A. 97:4520-4524.

19. Larsen, C.W., Hirst, E., Alexandre, C., and Vincent, J.P. 2003. Segment boundary formation in Drosophila embryos. Development. 130:5625-5635.

\title{
Altered regulation of IL-2 production in systemic lupus erythematosus: an evolving paradigm
}

Gary M. Kammer

Arthritis Associates Inc., Willoughby, Ohio, USA.

In systemic lupus erythematosus (SLE), IL-2 production by T lymphocytes in vitro is impaired. Deficient IL-2 production may be an outcome of a primary SLE T cell disorder that is due to impaired signal transduction. In this issue of the JCI, evidence is presented that an anti-TCR/CD3 complex autoantibody present in SLE sera can bind to $T$ cells and activate the $\mathrm{Ca}^{2+}$-calmodulin kinase IV (CaMKIV) signaling cascade, resulting in downregulation of $I L-2$ transcription and IL-2 production (see the related article beginning on page 996). Because IL-2 may contribute to the maintenance of T cell tolerance, deficient IL-2 production could promote a breach of $\mathrm{T}$ cell tolerance that results in autoantibody production in SLE.

Recently, it has been recognized that diverse autoantibodies directed against intra- and extracellular autoantigens exist in patients with systemic lupus erythematosus (SLE) for years before the clinical diagnosis is made (1); this suggests that physiologic mechanisms

Nonstandard abbreviations used: CaMKIV, $\mathrm{Ca}^{2+}$ calmodulin kinase IV; CRE, cAMP response element; CREB, CRE-binding protein; CREM, CRE modulator; IL-2R, IL-2 receptor; PCREB, phosphorylated CREB; pCREM, phosphorylated CREM; PKA-II $\beta$, type II $\beta$ PKA; pRII $\beta$, phosphorylated RII $\beta$; RII $\beta$, $\beta$ type II regulatory subunit; SLE, systemic lupus erythematosus.

Conflict of interest: The author has declared that no conflict of interest exists.

Citation for this article: J. Clin. Invest. 115:836-840 (2005). doi:10.1172/JCI200524791. that maintain tolerance to self antigens have been breached. Tolerance to self antigens is established and preserved by a subpopulation of T lymphocytes known as Tregs (2), and the loss of tolerance is a pathologic process giving rise to autoimmunity. This circumstance raises the possibility of the existence of abnormal $\mathrm{T}$ cell clones that mediate defective helper and suppressor effector functions, which result in autoantibody generation by forbidden $\mathrm{B}$ cell clones. In SLE, defective signaling cascades are believed to give rise to a primary $\mathrm{T}$ cell disorder that is characterized by impaired effector functions (3). These effector dysfunctions are, at least in part, a result of skewed expression of various effector molecules, including CD40 ligand (e.g., CD154) and multiple cytokines, and may reflect an imbalance of gene expression. An extracellular factor(s) in the microenvironment that interacts with $\mathrm{T}$ cells and exacerbates these dysfunctions has not been previously identified.

\section{Tregs, skewed cytokine production, and loss of tolerance}

Impaired effector $\mathrm{T}$ cell functions due to skewed cytokine production may create a microenvironment that promotes a strong Th2 immune response relative to Th1 and Treg activity. Relative overproduction of IL-4, IL- 6 , and IL- 10 by Th 2 cells and underproduction of IL-2, IL-12, TGF- $\beta$, and IFN- $\gamma$ by Th1 cells and Tregs can result in imbalanced autocrine and paracrine effects on $\mathrm{T}$ and $\mathrm{B}$ cells in the microenvironment. Because of the reduced numbers of $\mathrm{CD} 4{ }^{+} \mathrm{CD} 25^{+}$Tregs (4) as well as the diminished generation of IL-2 and TGF- $\beta$, there may be insufficient suppressor activity in SLE to counterbalance the enhanced Th2 effect on B cell antibody production. Taken together, these conditions create a microenvironment that promotes a 
Table 1

Abnormal signaling molecules in SLE T cells

\begin{tabular}{|c|c|c|c|}
\hline Pathway segment & Signaling molecule & Functional effect & References \\
\hline \multirow[t]{5}{*}{ Proximal } & CD45 tyrosyl phosphatase & Deficient activity & (19) \\
\hline & TCR $\zeta$ chain & Deficient/absent homodimer & $(20,21)$ \\
\hline & Lck tyrosyl kinase & Deficient & (22) \\
\hline & $\mathrm{IP}_{3}$ and $\mathrm{Ca}^{2+}$ & Increased $\mathrm{IP}_{3}$ turnover & \\
\hline & & $\begin{array}{l}\text { Increased, prolonged intracellular } \\
\mathrm{Ca}^{2+} \text { concentrations }\end{array}$ & (16) \\
\hline \multirow[t]{4}{*}{ Middle } & PKA-I and PKA-II isozymes & Deficient activities & $(18,23)$ \\
\hline & PKC & Deficiency & (24) \\
\hline & Mitochondria & $\begin{array}{l}\text { Abnormal hyperpolarization, } \\
\text { ROS, ATP depletion }\end{array}$ & $(25,26)$ \\
\hline & $\mathrm{Cbl}$ & Regulatory defect & (27) \\
\hline \multirow[t]{6}{*}{ Distal } & Elf-1 & Deficiency & (28) \\
\hline & AP-1 & Deficiency & (29) \\
\hline & P65-RelA subunit of NF- $\kappa B$ & Deficiency & (30) \\
\hline & pCREM & $\begin{array}{l}\text { Increased nuclear binding to }-180 \\
\text { site of IL-2 promoter/enhancer }\end{array}$ & (12) \\
\hline & Ras-MAPK & Deficiency & (31) \\
\hline & PKR expression & Impaired translation & (32) \\
\hline
\end{tabular}

AP-1, activator protein-1; $I P_{3}$, inositol triphosphate; PKR, dsRNA-regulated protein kinase. Modified with permission from Arthritis and Rheumatism (3).

dysregulated immune response driving both physiologic and forbidden B cell clones to overproduce antibodies and autoantibodies, which results in hypergammaglobulinemia. Moreover, these events occur despite the existence of other primary counterregulatory mechanisms, including expression of the cell surface molecule cytotoxic $T$ lymphocyte antigen 4 (CTLA-4) (5).

IL-2 is a key cytokine that has been held to function predominantly as a growth factor. This cytokine is largely produced by activated $\mathrm{CD} 4^{+}$and $\mathrm{CD} 8^{+} \mathrm{T}$ cells and binds to high-affinity cell surface IL-2 receptors (IL-2Rs) expressed by $\mathrm{T}$ cells, $B$ cells, NK cells, and APCs. However, current evidence from analyses of IL-2-/and IL-2R $\mathrm{R}^{-/}$knockout mice supports the notion that IL-2 may operate, not as a principal growth factor in vivo, but as a third signal that stimulates clonal expansion of effector cells to promote tolerogenic responses and to regulate development and function of $\mathrm{CD} 4{ }^{+} \mathrm{CD} 25^{+}$Tregs and, possibly, $\mathrm{CD}^{+}$Tregs to maintain tolerance $(6,7)$. Although much less is known about the mechanisms of IL-2 function in humans, it seems reasonable to suppose that IL-2 may serve a parallel role in immune homeostasis.

\section{Mechanisms of deficient IL-2 production by SLE T cells}

Deficient IL-2 production may predispose individuals to impaired immunoregula- tion, loss of tolerance, and the development of SLE owing to the abrogation of suppressor mechanisms that maintain tolerance to self antigens. Two lines of evidence support this concept. First, it has been demonstrated that $T$ cells from animal models of lupus as well as patients with SLE produce low amounts of IL-2 in vitro $(8,9)$. Second, vaccination of MRL/lpr lupus mice with live vaccinia recombinant viruses expressing the human IL-2 gene ameliorated disease activity (10). However, at the time that the IL-2 deficiency was discovered, the mechanisms leading to deficient IL-2 production by SLE T cells were unknown.

In SLE, a primary $\mathrm{T}$ cell disorder has been proposed to exist based on the identification of multiple discrete signaling abnormalities at the level of the TCR/CD3 complex, the cytosol, and the nucleus $(3,11)$ (summarized in Table 1). Tsokos, Kammer, and their colleagues first proposed that a primary failure of $\mathrm{T}$ cells due to defective signaling could hinder $I L$-2 gene transcription and IL-2 production and contribute to impaired $\mathrm{T}$ cell effector functions in SLE (12). To date, the data support this notion (13). Figure 1 presents a schematic of the mechanisms identified to date in SLE T cells that contribute to the downregulation of IL-2 production. However, extracellular factors in the microenviron ment that impinge on the SLE T cell to further modify its immunoregulatory functions could also exist.

\section{IgG anti-TCR/CD3 autoantibodies and reduced IL-2 generation}

In this issue of the JCI, Tsokos and colleagues present an incisive series of experiments that demonstrate that there is a factor in the microenvironment that can induce inhibition of IL-2 production by SLE $\mathrm{T}$ cells (14). Here, these authors show for what is believed to be the first time that the IgG fraction of SLE serum possesses an antiTCR/CD3 complex autoantibody(s) that stimulates translocation of $\mathrm{Ca}^{2+}$-calmodulin kinase IV (CaMKIV) from the cytosol to the nucleus. This event induces upregulation of the CREM (cAMP response element [CRE] modulator) transcript and CREM protein, proposed phosphorylation of CREM (to PCREM), binding of pCREM homodimers as well as pCREM-phosphorylated CREbinding protein (PCREB) heterodimers to the -180 region of the IL-2 promoter/ enhancer, and leads to reduced IL-2 transcription and IL-2 production (Figure 1).

It has not been previously shown that an IgG autoantibody in SLE serum can activate a signaling cascade in $\mathrm{T}$ cells, although it is well known that serum factors can activate signaling pathways in diverse cell types. Indeed, we have previously found that SLE serum does not induce the defects of PKA signaling in normal $T$ cells that have been identified in SLE T cells (15). To determine whether such an activating factor exists in SLE serum, Tsokos and his associates first investigated whether SLE sera could cause increased expression of nuclear pCREM relative to PCREB in normal $\mathrm{T}$ cells, just as had been observed in SLE T cells. Indeed, in normal $\mathrm{T}$ cells cultured in the presence of SLE sera, but not sera from patients with rheumatoid arthritis or from healthy controls, the authors observed increased levels of the CREM transcript, CREM protein, and PCREM binding to the - 180 CRE region of the $I L-2$ promoter/enhancer. These findings revealed that SLE sera contain a factor capable of inducing nuclear CREM expression (14).

In subsequent experiments, the authors identified the protein kinase that mediates the overexpression of CREM. Based on their previous recognition that signals from the TCR/CD3 complex significantly stimulate the production of increased concentrations of intracellular $\mathrm{Ca}^{2+}$ in SLE T cells (16), they searched for a $\mathrm{Ca}^{2+}$-dependent kinase and found that SLE T cells exhibited increased amounts of nuclear CaMKIV compared to nuclear CaMKII in 


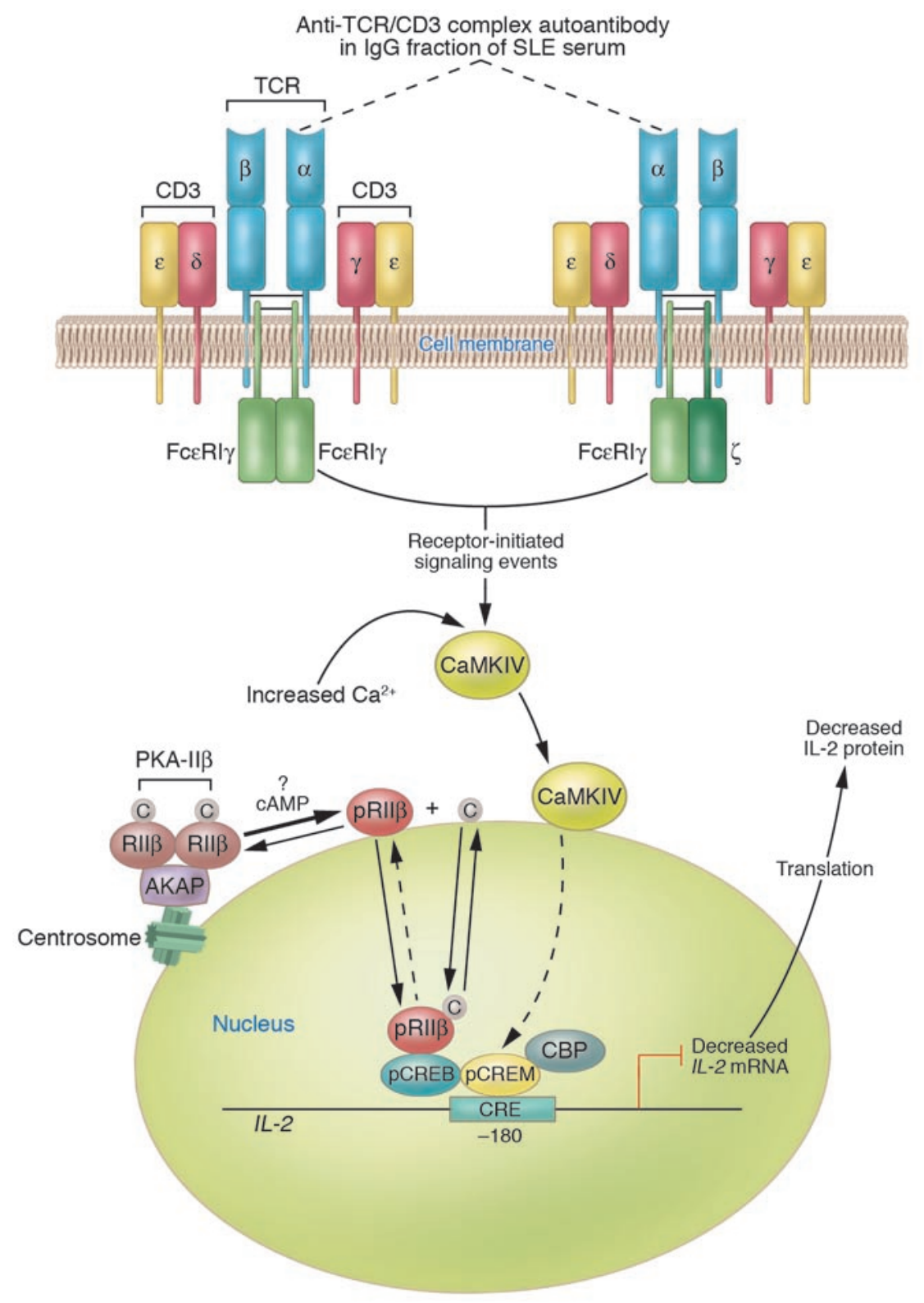

normal $\mathrm{T}$ cells. When normal $\mathrm{T}$ cells were then incubated with SLE or normal control sera, transiently transfected with a CaMKIV expression plasmid, and activated with a phorbol ester and ionomycin, T cells exposed to SLE sera exhibited significantly greater pCREM binding to the -180 region of the IL-2 promoter/enhancer (14). When these $\mathrm{T}$ cells were also transiently transfected with an $I L-2$ promoter-reporter luciferase construct, the cells incubated with SLE sera expressed significantly lower $I L-2$ promoter luciferase activity and IL-2 production compared with cells incubated in normal sera. Taken together, these results provide firm evidence that an extracellular factor in SLE sera can activate
CaMKIV and induce its nuclear translocation from the cytosol, where it upregulates CREM expression, pCREM binding to the $I L-2$ promoter/enhancer, and inhibition of IL-2 transcription and IL-2 production.

To identify the SLE serum factor, the authors postulated that the TCR/CD3 complex is the target for a circulating IgG autoantibody(s) in SLE serum. They performed a subtraction experiment using wild-type and mutant Jurkat T cells that do not express the surface TCR/CD3 complexes. Absorption of the IgG autoantibody(s) by wild-type Jurkat $\mathrm{T}$ cells but not TCR/ CD3- mutant Jurkat $T$ cells eliminated the ligand(s) for the TCR/CD3 complex, reducing CaMKIV nuclear translocation

\section{Figure 1}

Diagram of established and proposed mechanisms contributing to reduced IL-2 production by SLE T cells. A primary T cell disorder characterized by multiple abnormal signaling molecules has been identified in human SLE. A proportion of TCR $\zeta$ chains are replaced with $\mathrm{Fc \varepsilon}_{\mathrm{C}} \mathrm{Rl}$ chains through which signaling can take place. In the microenvironment, antiTCR/CD3 autoantibody(s) can bind to SLE T cells via the TCR/CD3 complex; however, the subunit(s) of the TCR/CD3 complex against which the autoantibody(s) is directed has not yet been identified. For the purpose of this illustration, the autoantibody is proposed to be against the $\alpha$ subunit of the TCR (dotted lines). Autoantibody binding to the TCR/CD3 complex results in heightened intracellular $\mathrm{Ca}^{2+}$ concentrations and activation of the CaMKIV signaling cascade. CaMKIV translocates to the nucleus, is proposed to phosphorylate CREM, and induces increased pCREM binding to the CRE located at the -180 region of the $I L-2$ promoter/enhancer. Because there is overexpression of the RII $\beta$ in the $T$ cell nuclei of some patients with SLE, it is proposed that PKA-II $\beta$ is spontaneously activated by an unknown mechanism and that RII $\beta$ becomes autophosphorylated; $p R I I \beta$ translocates to the nucleus, where it mediates downregulation of $I L-2$ transcription and IL-2 production. Together, pCREM and $p R I I \beta$ inhibit IL-2 transcription, thus yielding reduced IL-2 production. Note that proposed mechanisms have not been experimentally demonstrated. C, catalytic subunit; CBP, CREB binding protein. and PCREM binding to the IL-2 promoter/ enhancer (14). Although the authors did not perform an experiment to identify the subunit of the TCR/CD3 complex against which the autoantibody(s) is directed, the results support the notion that SLE sera possess an anti-TCR/CD3 complex autoantibody(s) that can bind to a subunit(s) of the TCR/ CD3 complex and activate the CaMKIV signaling cascade in T cells (Figure 1).

Movement of signaling molecules between intracellular compartments is a well-known concept in cell biology. Under homeostatic conditions, signaling molecules are localized to specific intracellular regions; in response to a stimulus, these molecules can translocate 
to a target to perform a specific function, such as phosphorylation. cAMP-dependent activation of type II $\beta$ PKA (PKA-II $\beta$ ) releases the $\beta$ type II regulatory subunit (RII $\beta$ ) from its binding site on A-kinase anchor proteins (AKAPs) and its interaction with the catalytic subunit, permitting its autophosphorylation and translocation from the cytosol to the nucleus in $\mathrm{T}$ cells (17) (Figure 1). Once in the nucleus, phosphorylated RII $\beta$ (pRII $\beta$ ) binds to $\mathrm{PCREB}$, inhibits activator protein-1 formation, and strongly inhibits $I L-2$ transcription and IL-2 production. In SLE, the accumulation of RII $\beta$ in the nuclei of $\mathrm{T}$ cells from some patients seems likely to contribute to reduced IL-2 production (18) (Figure 1). The identification of CaMKIV in the nuclei of SLE $T$ cells is the second example of cytosolto-nuclear translocation of a protein kinase. Despite our incomplete understanding of the repressor mechanisms involved at the level of the basal transcription machinery, the recognition that both nuclear CaMKIV and pRII $\beta$ have the effect of downregulating IL-2 production suggests complementary functions with a powerful outcome $(14,17)$.

The weight of evidence to date has led investigators to propose that the signaling abnormalities previously identified in SLE $\mathrm{T}$ cells reflect a disorder primary to the $\mathrm{T}$ cell $(3,11)$. Here, Tsokos and his colleagues present the first credible evidence that an extracellular factor(s) in the microenvironment can trigger a signaling cascade that ultimately modulates $I L-2$ transcription in T cells (14). It may be that extracellular factors further hinder an already impaired $\mathrm{T}$ cell by activating functional pathways via cell surface receptors.

\section{Reduced IL-2 production, effector and Treg functions, and tolerance in SLE}

Two fundamental concepts seem to be emerging from the IL-2-/- and IL-2 $\mathrm{R}^{-/-}$ knockout murine models that may become applicable to the study of SLE in humans. The first is that IL-2 is necessary for effective Treg maintenance of homeostasis via mechanisms involving anergy and apoptosis. Loss of IL-2 in the murine system is associated with reduced numbers of $\mathrm{CD} 4{ }^{+} \mathrm{CD} 25^{+} \mathrm{T}$ cells and autoimmunity; restoration of IL-2 prevents autoimmunity and generates homeostasis. The second is that IL-2 may operate as a third signal for the expansion, develop- ment, and function of antigen-dependent $T$ cell effectors $(6,7)$.

In human SLE, reduced IL-2 generation may be a key factor underlying the impaired $\mathrm{T}$ cell effector functions and breach of tolerance that result in autoantibody production. In the murine system, development of CD $4^{+} \mathrm{CD} 25^{+}$Tregs in the thymus and their subsequent export to and expansion in the periphery are dependent on IL-2 (7). Whether there is a similar requirement for IL-2 in the development and expansion of $\mathrm{CD} 4{ }^{+} \mathrm{CD} 25^{+}$ Tregs in humans remains uncertain $(6,7)$. Although there is still limited information about $\mathrm{CD} 4{ }^{+} \mathrm{CD} 25^{+}$Treg function in human SLE, one might hypothesize that the reduced numbers of these Tregs in the periphery in SLE (4) reflect a defect in their development, export, and/or proliferation in the periphery.

\section{Future directions}

Clearly there is a paucity of information about IL-2 function in human immunity and tolerance, the molecular mechanisms regulating its expression in $\mathrm{T}$ cells, and its contribution to the immunopathogenesis of SLE. This gap in knowledge presents a compelling opportunity to investigate these issues. Among possible future directions, establishing the mechanisms of IL-2 regulation of homeostasis in humans should generally be emphasized. Because there is consensus that a breach of $\mathrm{T}$ cell tolerance contributes to the persistence of autoreactive T cells and autoantibody production, mechanisms that may result in this failure should be intensively investigated. In this vein, the contribution by Tsokos and his colleagues in this issue of the JCI (14) provides a new appreciation and insight into how the microenvironment in SLE can further impinge on a defective $\mathrm{T}$ cell to inhibit IL-2 production. From such studies will come the inspiration and novel approaches necessary to develop therapeutic tools to abate disease and improve the quality of life of our patients.

Address correspondence to: Gary M. Kammer, Arthritis Associates Inc., 36100 Euclid Avenue, Suite 260, Willoughby, Ohio 44094, USA. Phone: (440) 953-8700; Fax: (440) 9538796; E-mail: gmkammer@hotmail.com.

1. Arbuckle, M.R., et al. 2003. Development of autoantibodies before the clinical onset of systemic lupus erythematosus. N. Engl. J. Med. 349:1526-1533.

2. Thompson, C., and Powrie, F. 2004. Regulatory T cells. Curr. Opin. Pharmacol. 4:408-414.
3. Kammer, G.M., Perl, A., Richardson, B.C., and Tsokos, G.C. 2002. Abnormal T cell signal transduction in systemic lupus erythematosus. Arthritis Rheum. 46:1139-1154.

4. Liu, M.F., Wang, C.-R., Fung, L.-L., and Wu, C.-R 2004. Decreased CD4+CD25+ T cells in peripheral blood of patients with systemic lupus erythematosus. Scand. J. Immunol. 59:198-202.

5. Jiang, H., and Chess, L. 2004. An integrated view of suppressor $\mathrm{T}$ cell subsets in immunoregulation. J. Clin. Invest. 114:1198-1208. doi:10.1172/ JCI200423411.

6. Nelson, B.H. 2004. IL-2, regulatory T cells, and tolerance. J. Immunol. 172:3983-3988.

7. Malek, T.R., and Bayer, A.L. 2004. Tolerance, not immunity, crucially depends on IL-2. Nat. Rev. Immunol. 4:665-674.

8. Wofsy, D., et al. 1981. Deficient interleukin 2 activity in MRL/Mp and C57BL/6J mice bearing the lpr gene. J. Exp. Med. 154:1671-1680.

9. Alcocer-Varela, J., and Alarcon-Segovia, D. 1982. Decreased production of and response to interleukin-2 by cultured lymphocytes from patients with systemic lupus erythematosus. J. Clin. Invest. 69:1388-1392.

10. Gutierrez-Ramos, J.C., Andreu, J.L., Revilla, Y., Vinuela, E., and Martinez, C. 1990. Recovery from autoimmunity of MRL/lpr mice after infection with an interleukin-2/vaccinia recombinant virus. Nature. 346:271-274.

11. Dayal, A.K., and Kammer, G.M. 1996. The T cell enigma in lupus. Arthritis Rheum. 39:23-33.

12. Solomou, E.E., Juang, Y.-T., Gourley, M.F., Kammer, G.M., and Tsokos, G.C. 2001. Molecular basis of deficient IL-2 production in T cells from patients with systemic lupus erythematosus. J. Immunol. 166:4216-4222.

13. Kyttaris, V.C., and Tsokos, G.C. 2004. T lymphocytes in systemic lupus erythematosus: an update. Curr. Opin. Rheumatol. 16:548-552.

14. Juang, Y.-T., et al. 2005. Systemic lupus erythematosus serum IgG increases CREM binding to the $I L-2$ promoter and suppresses IL-2 production through CaMKIV. J. Clin. Invest. 115:996-1005. doi:10.1172/ JCI200522854.

15. Kammer, G.M., Haqqi, T.M., Hasler, P., and Malemud, C.J. 1993. The effect of circulating serum factors from patients with systemic lupus erythematosus on protein kinase A (PKA) activity and PKA-dependent protein phosphorylation in T lymphocytes. Clin. Immunol. Immunopathol. 67:8-16.

16. Vassilopoulos, D., Kovacs, B., and Tsokos, G.C. 1995. TCR/CD3 complex-mediated signal transduction pathway in $\mathrm{T}$ cells and $\mathrm{T}$ cell lines from patients with systemic lupus erythematosus. J. Immunol. 155:2269-2281.

17. Elliott, M.R., et al. 2004. Down-regulation of IL-2 production in $\mathrm{T}$ lymphocytes by phosphorylated protein kinase A-RIIb. J. Immunol. 172:7804-7812.

18. Mishra, N., Khan, I.U., Tsokos, G.C., and Kammer, G.M. 2000. Association of deficient type II protein kinase A activity with aberrant nuclear translocation of the RIIb-subunit in systemic lupus erythematosus T lymphocytes. J. Immunol. 165:2830-2840.

19. Takeuchi, T., Pang, M., Amano, K., Koide, J., and Abe, T. 1997. Reduced protein tyrosine phosphatase (PTPase) activity of CD45 on peripheral blood lymphocytes in patients with systemic lupus erythematosus. Clin. Exp. Immunol. 109:20-26.

20. Liossis, S.N.C., Ding, X.Z., Dennis, G.J., and Tsokos, G.C. 1998. Altered pattern of TCR/CD3-mediated protein-tyrosyl phosphorylation in T cells from patients with systemic lupus erythematosus. Deficient expression of the $\mathrm{T}$ cell receptor zeta chain. J. Clin. Invest. 101:1448-1457.

21. Takeuchi, T., et al. 1998. TCRz chain lacking exon 7 in two patients with systemic lupus erythematosus. Int. Immunol. 10:911-921.

22. Matache, C., et al. 1999. p56lck activity and expres- 
sion in peripheral blood lymphocytes from patients with systemic lupus erythematosus. Autoimmunity. 29:111-120.

23. Kammer, G.M., Khan, I.U., and Malemud, C.J. 1994. Deficient type I protein kinase A isozyme activity in systemic lupus erythematosus $\mathrm{T}$ lymphocytes. J. Clin. Invest. 94:422-430.

24. Tada, Y., Nagasawa, K., Yamauchi, Y., Tsukamoto, H., and Niho, Y. 1991. A defect in the protein kinase $\mathrm{C}$ system in $\mathrm{T}$ cells from patients with systemic lupus erythematosus. Clin. Immunol. Immunopathol. 60:220-231.

25. Gergely, P., Jr., et al. 2002. Persistent mitochondrial hyperpolarization, increased reactive oxygen intermediate production, and cytoplasmic alkalinization characterize altered IL-10 signaling in patients with systemic lupus erythematosus. J. Immunol. 169:1092-1101.
26. Gergely, P., Jr., et al. 2002. Mitochondrial hyperpolarization and ATP depletion in patients with systemic lupus erythematosus. Arthritis Rheum. 46:175-190.

27. Yi, Y., McNerney, M., and Datta, S.K. 2000. Regulatory defects in $\mathrm{Cbl}$ and mitogen-activated protein kinase (extracellular signal-related kinase) pathways cause persistent hyperexpression of CD40 ligand in human lupus T cells. J. Immunol. 165:6627-6634.

28. Juang, Y.-T., Tenbrock, K., Nambiar, M.P., Gourley, M.F., and Tsokos, G.C. 2002. Defective production of functional $98-\mathrm{kDa}$ form of Elf-1 is responsible for the decreased expression of TCR z-chain in patients with systemic lupus erythematosus. J. Immunol. 169:6048-6055.

29. Kyttaris, V.C., Juang, Y.-T., Tenbrock, K., Weinstein, A., and Tsokos, G.C. 2004. Cyclic adenosine 5 '-monophosphate response element modula- tor is responsible for the decreased expression of c-fos and activator protein-1 binding in T cells from patients with systemic lupus erythematosus. J. Immunol. 173:3557-3563.

30. Wong, H.K., Kammer, G.M., Dennis, G., and Tsokos, G.C. 1999. Abnormal NF-kB activity in T lymphocytes from patients with systemic lupus erythematosus is associated with decreased p65-RelA protein expression. J. Immunol. 163:1682-1689.

31. Deng, C., et al. 2001. Decreased ras-mitogen-activated protein kinase signaling may cause DNA hypomethylation in T lymphocytes from lupus patients. Arthritis Rheum. 44:397-407.

32. Grolleau, A., Kaplan, M.J., Hanash, S.M., Beretta, L., and Richardson, B.C. 2000. Impaired translational response and increased protein kinase PKR expression in T cells from lupus patients. J. Clin. Invest. 106:1561-1568.

\title{
In hypertension, the kidney is not always the heart of the matter
}

\author{
Michael E. Mendelsohn
}

Molecular Cardiology Research Institute, Tufts-New England Medical Center, Boston, Massachusetts, USA.

\begin{abstract}
Blood pressure abnormalities are thought to originate from intrinsic changes in the kidney, a concept that has been largely unchallenged for more than 4 decades. However, recent molecular, cellular, and transgenic mouse studies support an alternative hypothesis: primary abnormalities in vascular cell function can also directly cause abnormalities of blood pressure. In this issue of the JCI, Crowley and coworkers describe the application of an elegant cross-renal transplant model to type $1 \mathrm{~A}$ angiotensin $\left(\mathrm{AT}_{1 \mathrm{~A}}\right)$ receptor-deficient mice and their wild-type littermates to explore the relative contributions of renal and extrarenal tissues to the low blood pressure seen in the $\mathrm{AT}_{1 \mathrm{~A}}$ receptor-deficient animals (see the related article beginning on page 1092). Their studies further support the emerging paradigm that primary abnormalities of the vasculature can make unique, nonredundant contributions to blood pressure regulation; the findings have potentially important implications for the ways we diagnose and treat blood pressure diseases in humans.
\end{abstract}

\section{Introduction}

Abnormalities of blood pressure, especially high blood pressure or hypertension, are widespread, cause extensive morbidity and mortality, and are insidious, because they rarely generate symptoms. But what causes hypertension? This simple question is remarkably difficult to answer, in large

Nonstandard abbreviations used: $\mathrm{AT}_{1 \mathrm{~A}}$, type $1 \mathrm{~A}$

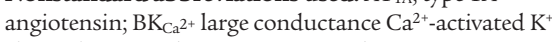
channel; $\mathrm{CO}$, cardiac output; $\mathrm{D}^{+} \mathrm{R}^{+}$, donor ${ }^{+}$recipient ${ }^{+}$; $\mathrm{IP}_{3}$, inositol triphosphate; MLC, myosin light chain; MLCK, MLC kinase; PKGI, cGMP-dependent protein kinase type I; PP1M, myosin phosphatase; RAS, reninangiotensin system; RGS2, regulator of G protein signaling 2; SVR, systemic vascular resistance.

Conflict of interest: The author has declared that no conflict of interest exists.

Citation for this article: J. Clin. Invest. 115:840-844 (2005). doi:10.1172/JCI200524806. part because blood pressure, which is measurable only in intact animals, is an integrated value determined by contributions from a complex mix of rheologic, renal, neural, vascular, and hormonal variables. It is therefore difficult to distinguish primary or causal factors for abnormal blood pressure from those responses that are secondary to the changes in pressure.

Blood pressure is proportional to 2 fundamental hemodynamic factors: cardiac output $(\mathrm{CO})$ and systemic vascular resistance $(\mathrm{SVR})$, and the equation $\mathrm{BP}=\mathrm{CO} \times \mathrm{SVR}$ is taught to every first-year physiology student. The pioneering studies of Guyton and colleagues provide abundant support for the central, causal role of abnormal renal sodium handling in the etiology of hypertension $(1,2)$. This work emphasiz- es the primacy of the kidney's "pressure control system," which is recruited when systemic pressure is altered and causes a renally mediated increase or decrease in blood volume to establish long-term blood pressure control. Elegant genetic studies published over the past decade have provided further strong support for the hypothesis that alterations of net salt balance underlie abnormalities of blood pressure in humans (3). But are the alterations in renal salt balance that cause abnormal blood pressure always direct, as has been argued for many years, or could renal alterations in some cases be indirect or secondary to primary abnormalities of nonrenal tissues? Does the kidney always cause abnormal blood pressure by altering blood volume, which in turn alters $\mathrm{CO}$ and SVR? Or do intrinsic abnormalities of the vasculature in some cases give rise to a primary abnormality of SVR, causing hypertension? Surprisingly, we do not yet know the answer to this simple dilemma.

In this issue of the JCI, Crowley and colleagues (4) describe cross-renal transplantation studies with type $1 \mathrm{~A}$ angiotensin $\left(\mathrm{AT}_{1 \mathrm{~A}}\right)$ receptor-deficient $\left(\right.$ Agtrla $\left.\mathrm{a}^{-/-}\right)$mice and their wild-type littermates. Using stateof-the-art approaches to examine mouse physiology and sophisticated, technically demanding microsurgical techniques, their study provides new and important insights into the relative importance of renal and nonrenal $\mathrm{AT}_{1}$ receptors in deter- 